\title{
A Framework for Personalized Fully Immersive Virtual Reality Learning Environments with Gamified Design in Education
}

\author{
Andreas MAROUGKAS ${ }^{1}$, Christos TROUSSAS, Akrivi KROUSKA and Cleo \\ SGOUROPOULOU \\ Department of Informatics and Computer Engineering, University of West Attica, \\ Greece
}

\begin{abstract}
Traditional learning methods frequently fail to provoke students' interest, stimulate their enjoyment and encourage them to participate in learning activities, resulting in discomfort, distractions, and disengagement, if not quitting. Education's goal is to improve the quality and effectiveness of teaching and learning methods. This paper aims to present a framework based on Virtual Reality (VR) technology and contemporary Head Mounted Displays, that incorporates game-based techniques and adaptive design according to the student's profile. As a result, this paper analyzes the relevant literature, the VR apparatus, the importance of VR, as well as gamification, personalization and adaptive design in education, which are the learning foundations on which the framework is based. Finally, the framework's modules and structure are presented, taking into account all of the previously mentioned parameters. This novel framework aspires to serve as a basis for educational applications that use immersive Virtual Reality technologies to transform learning procedures into entertaining, engaging, enjoyable, and effective experiences.
\end{abstract}

Keywords. Virtual Reality, framework, Virtual Reality Learning Environments , personalization, gamification

\section{Introduction}

Education is becoming a more demanding and complex field over the years with educators trying to deliver more efficient ways of transferring knowledge to the class. Traditional teaching methods have many disadvantages towards students. Educators have tested, researched and employed a wide variety of methods and platforms to facilitate educational approaches such as social networks [1,2], desktop computers [3], smartphones and tablets [4], Augmented Reality (AR) [5], mobile applications and online platforms [6].

The technological advancements of the last decade led researchers and educators to explore alternative methods of teaching students by taking advantage of new upcoming technologies. Virtual Reality (VR), Mixed Reality (MR) [7] and Augmented Reality

\footnotetext{
${ }^{1}$ Corresponding Author; E-mail: amarougkas@uniwa.gr.
} 
(AR) [8] are very promising approaches for an effective way of teaching and learning trying to solve various problems. Disengagement, lack of interest and enjoyment, reduced participation, discomforts and distractions are only a few of the problems that lead to students' poor learning outcomes and false perceptions about the subject of learning. VR made a giant leap from passive learning methods to more interactive experiences that students find useful, exciting, approachable, enjoyable, interesting and engaging [9].

After decades of failed attempts to be established in tech community, the hardware limitations related to severe oculomotor malfunctions, such as nausea and vertigo, have finally be solved. Smartphones can be incorporated with cardboard headsets delivering an affordable alternative access to VR applications. But the most noteworthy hardware is the Head Mounted Displays that offer fully immersive experiences accompanied by hardware controllers, hand tracking and gesture recognition. They also offer high visual fidelity, option to use it as a stand-alone wireless device or to connect it with a computer for more complex graphics. Contemporary HMDs offer a new path to education where the student is offered active participation in the learning process and a hands-on experience.

VR provides a new fresh, exciting and effective method of learning [10] for students, but sometimes that is not enough for students to capture their interest across the learning process. As an innovative approach to adaptive learning, this paper presents a framework that incorporates gamification design and personalization techniques into VR environments. Although gamified VR applications have been successfully employed, there is a lack of incorporating adaptive learning design in gamified Virtual Reality Learning Environments (VRLEs). It is essential to take into consideration students' unique characteristics and abilities to diversify the learning process creating a dynamic system serving students' academic requirements [2].

\section{Relevant work}

The improvements in VR hardware throughout the last decade have led many researchers to reformulate Virtual Environments for educational purposes and to further explore the potential of VR in the field of education. In certain cases, VR has been used passive learning [11]; while in other cases, the student is offered the opportunity of integrating with the virtual world as part of the learning $[12,13]$.

Cochrane et al. [14] proposed a design-based research (DBR) framework for implementing VRLEs to mobile devices. Furthermore, they presented two ongoing projects for higher education use as examples.

The findings of a thorough review in relevant studies led us to the conclusion that the most notable approaches to VR regarding education incorporate game-based logic and personalization techniques. Even if a VRLE is generally effective in terms of achieving the desired educational outcomes, this does not indicate that students perceive it to be an appealing learning method [15]. As a result, the willingness to use it and acknowledge it as a learning method could be negatively impacted, potentially leading to disengagement during the learning process.

Mapping gamification techniques to educational applications is a way to pique students' interest, engage and motivate them in the learning process. Hung et al. [16] developed a Digital Game-Based Learning (DGbL) environment for elementary school mathematics based on e-books. Although the application was not supported by a VR 
approach, it demonstrates the effectiveness of implementing gamification strategies in Learning Environments with immersive technologies.

Rychkova et al. [13] presented "Orbital Battleship", a game-based VR application that combined educational content in the field of chemistry with multiplayer gameplay. The application produced beneficial results for students, exceeding the authors' expectations. Despite the gamification techniques and a fully immersive Head Mounted Display used, as well as the positive learning outcomes emerged, the application was not designed to provide students with an adaptive experience that could be dynamically adjusted to their academic needs.

Wilson et al. [17] evaluated a VR ophthalmoscopy training application using gamification techniques, such as in-app, virtual awards to capture students' attention and keep them engaged throughout the procedure. Students felt a sense of accomplishment, which is one of the intrinsic factors that can lead to student retention and engagement. Despite the fact that the application provided methods for personalizing the learning process, the system lacked adaptivity in accordance with the academic requirements of the students.

Zhang et al. [18] created a VR simulation for fire safety education. Through their exploration of the virtual environment, students were given the ability to interact with virtual elements in order to construct their knowledge. The authors applied gamification techniques to captivate students' interest, such as reward strategies, that made the procedure appear both challenging and appealing. Through interactions, students received personalized feedback on learning errors, allowing them to learn more effectively. However, due to the subject's nature, it did lack adaptive techniques that could target students' learning needs and personal preferences.

\section{Virtual Reality as a medium}

Virtual Reality is built around a computer-generated space that enables the user to participate in a three-dimensional simulation while feeling immersed and experiencing realism. The element of interaction between the user and the virtual environment is a fundamental aspect of VR. Over the years, researchers and developers have used the term "Virtual Reality" to refer to a wide range of hardware devices such as CAVE VR [19], Desktop VR [3], Mobile VR [20], Head Mounted Device VR [13] glasses using stereoscopic imaging technology [21] and more. During the last decade, VR finally laid the groundwork for mass acceptance. After years of research and development, companies, such as HTC and Oculus, made Head Mounted Display devices accessible to the public through retail versions, which are now intertwined with the definition of VR. HMDs are now a well-established type of hardware that is widely available on the market.

Head-Mounted Displays efficiently transport the user from the physical to the virtual world by combining visual simulations, sounds and real-time interactions with virtual objects. Users currently employ two types of VR headsets depending on their requirements and preferences: hardware systems, such as the Oculus Quest and HTC Vive, and Cardboards, which use smartphone devices.

Each specific device is chosen based on what users evaluate that it is appropriate in each unique circumstance, with each having its own set of key advantages and disadvantages, as shown in Table 1. 
Table 1. An apparatus comparative chart

\begin{tabular}{lll}
\hline & \multicolumn{1}{c}{ Hardware HMD's } & \multicolumn{1}{c}{ Cardboards } \\
\hline Advantages & Higher visual fidelity & Easy access with any smartphone device \\
& $\begin{array}{l}\text { Six Degrees of Freedom (6-DoF) } \\
\text { provide movement flexibility }\end{array}$ & Cost effective for group use applications \\
& $\begin{array}{l}\text { Better interaction experience with } \\
\text { virtual environment with to two } \\
\text { advanced controllers }\end{array}$ & \\
Limitations & $\begin{array}{l}\text { Requires acquisition of new device } \\
\text { High costs for group use applications }\end{array}$ & $\begin{array}{l}\text { Three Degrees of Freedom (3-DoF) } \\
\text { provide limited movement flexibility }\end{array}$ \\
& & Lower visual fidelity
\end{tabular}

\section{Virtual Reality in education}

\subsection{Virtual Reality as a Virtual Learning Environment}

Throughout the last decade, Virtual Reality has gradually attracted more educators and researchers to work on projects demonstrating that it is a promising teaching and learning tool. Despite the fact that it is still under-researched technological approach in terms of its potential, it has already been implemented in various fields of education such as architecture [22], biology [20] chemistry [23], engineering [10], health sciences [17], physics [24], religion [25] and video game development [26].

When compared to traditional Virtual Learning Environments, Virtual Reality Learning Environments (VRLE) are becoming a more effective and appealing alternative. VRLEs have been used mostly in desktop computers for decades as part of the learning process, being limited in terms of interaction and field of view. They make use of a keyboard, mouse, and monitor, but VRLEs make use of advanced controllers or even hand gestures. VRLEs also provide a broader field of view with no framed boundaries, resulting in a more intuitive sense of freedom, interaction and perspective within the artificial environment, promoting a "Learning-by-doing" experience [27].

\subsection{Benefits}

In accordance with the "Flow Theory", VR immerses users in the experience, while their attention is completely absorbed within the virtual simulation [28]. With contemporary VR headsets, users can achieve high levels of immersion and embodiment. Using controllers, they interact in real-time with the environment, transforming the overall experience into an effective aid to students' learning and developing a sense of being there. VR allows users view realistic portraits of real-world environments, situations and objects, enhancing their spatial presence [11].

VR applications in education, taking into account all of the aforementioned properties, can have a positive impact on a variety of aspects of students' perceptions, like perceived learning motivation, which encourages them to participate in related activities [9]. Furthermore, the intention to use it increases enjoyability because students find it appealing [29]. 
Moreover, students lose interest in traditional in-class learning events, resulting in a circumstance in which their educational results are harmed. Students' interest can be captivated when VR opens up a previously uncharted territory, encouraging them to not only participate more than they normally would, but also to be more involved in the learning process, which can lead to improved learning outcomes [11].

VR has a high didactic and practical value, and when used in conjunction with traditional methods, it has the potential to improve students' understanding [29], assist them to master knowledge [30] and produce better learning outcomes [31]. It may also enable students to carry out curriculum-related experiments and procedures that would otherwise be prohibitively expensive, dangerous, or impossible [32].

\subsection{Limitations}

Despite the benefits and potential of using VR as a teaching or learning method, some significant limitations must be considered. For example, despite the limited amount of time the user operated the system, some oculomotor-related symptoms such as nausea have been reported in some cases [12]. Dizziness, vertigo, eye strain, and headaches are also symptoms of users' sensory conflict. Therefore, it is critical to check users' backgrounds before involving them in any VR-related process, due to cybersickness symptoms [33].

\section{Gamification}

\subsection{A gamified classroom}

When compared to commercial video games, in-class learning activities reveal similar behavioral patterns among students and gamers [34]. For example, both allow students to prioritize their tasks, as in turn-based role-playing games, design and develop strategies in the same way as in real-time strategy games. Moreover, they can develop new skills and improve old ones through practice, such as in sports games; learn to be adaptable and creative in new situations by playing adventure games; develop critical thinking skills, as in puzzle games; learn to be persistent, patient and goal-oriented, just like in action role-playing games and in multiplayer and online games, where players can change their gaming style to adjust to group activities.

Gamification manages to combine commercial games and education by employing game design, game mechanics, and techniques in non-gaming contexts of the curriculum to improve students' learning experiences by making them quite enjoyable and engaging. This can help to solve the problem of students' lack of interest in getting involved in school activities by increasing their motivation and interest in the relevant curricula.

\subsection{Gamification and Virtual Reality in education}

The logic, techniques, and mechanics of video games can be successfully implemented in the frameworks of Virtual Reality Learning Environments to create simulations based on educational content.

Students can interact with the artificial environment and interfere in assigned tasks designed according to their educational needs using VR controllers, which are 
individually created for each head-mounted display system, just as they would do in video game quests of commercial games using ordinary video gaming controllers.

When combined with gamified features, VR educational content that focuses on student motivation and engagement can lead to better learning outcomes [18], improve memory retention [33] and enhance content knowledge [35]. Gamification techniques of various types can be incorporated into Virtual Reality Learning Environments for educational purposes. Live frequent updates on information about students' scores, ongoing state, progress status, and milestones achieved in each stage are among the features that can assist students throughout the VR learning process. Furthermore, rewarding students with badges for game-like achievements, and leaderboards as a projection of ranking based on their scores [15] are both effective methods of improving student retention and enhancing their excitement. Students can also collaborate or compete in a multiplayer mode [13]. A help support repository in the menu, different levels of difficulty, objectives, puzzles, and item inventories are also gamification techniques.

\section{Personalized Virtual Learning Environments}

An important way to support a virtual learning environment efficiently is to use personalization techniques with the goal of delivering a tailored experience based on student profiling. This can be accomplished by taking into account students' preferences, interests, needs, capabilities, skills, prior knowledge of the subject, learning styles, and even special needs, thereby addressing the challenges of the heterogeneous features of twenty-first-century education. A personalized Virtual Learning Environment can benefit students by assisting them in improving their content knowledge, learning outcomes, and academic achievements. It also eliminates any perceptual discomforts that may arise during the learning process, such as cognitive load, frustration, or a lack of interest. These discomforts may act as impediments to learning, resulting in ineffective or distorted learning outcomes, or even quitting.

To clear up any confusion, a personalized learning environment is an adaptive automated system based on individualization, as opposed to a customizable environment, which requires manual adjustments before it can be applied to any educational intervention.

Various techniques and theories can be used to compose a model design with flexible modules, dynamic data filtering, data logging for registering behavioral parameters and preferences, and thus creating an application based on the cognitive diversity and mentality of each student [36].

Troussas et al. [6] presented a model of learning analytics regarding learning environments which included multiple modules such as cognitive state and behavior prediction module, identification of targeted educational material module, curriculum improvement module and personalization module. The findings of this study, which included students' evaluations of a web-based prototype application based on the aforementioned modules, were very promising, as students perceived the system as an efficient way to participate in a personalized experience.

Regarding learning analytics in VR, Srimadhaven et al. [37] proposed an architecture of a VR mobile game for undergraduate students related to the subject of programming languages. In this project, students had to complete three stages of the game by performing coding tasks. Each level provided a different level of difficulty, 
allowing students to earn points based on question correctness. The authors designed the system's architecture to assess students' cognitive behavior, self-regulation, affective behavior, and self-efficacy in order to improve students' academic performance, with learning analytics assisting the entire process by providing a personalized experience based on student results individualization.

\section{A proposed framework}

After the thorough literature review presented above, we came up with the conclusion that the integration of VR Learning Environments with personalized gamified mechanisms that take into account students' cognitive states and behavioral patterns while also balancing gameplay and learning objectives has never been approached with fully immersive VR Head Mounted Displays as medium hardware. It is critical to identify students' learning gaps in order to provide a tailored solution that not only meets his/her needs and competencies [38], but also to pique his/her interest and increase excitement, resulting in an engaged and overall enjoyable experience.

The following proposed framework (as shown in Fig. 1) aspires to be a reference point for future research and educational designs, enabling the inclusion of all of the above-mentioned approaches. The framework is comprised of the pedagogical design, which includes the formulation of educational objectives, learning theories, and strategies as part of the student-centered approach. The Technological Design includes all of the technological mechanisms, software, and hardware required to connect the theoretical design to the artificial environment. The Data Collection Layer is responsible for the generation, storage, and retrieval of data produced by the user's interactions with the system.

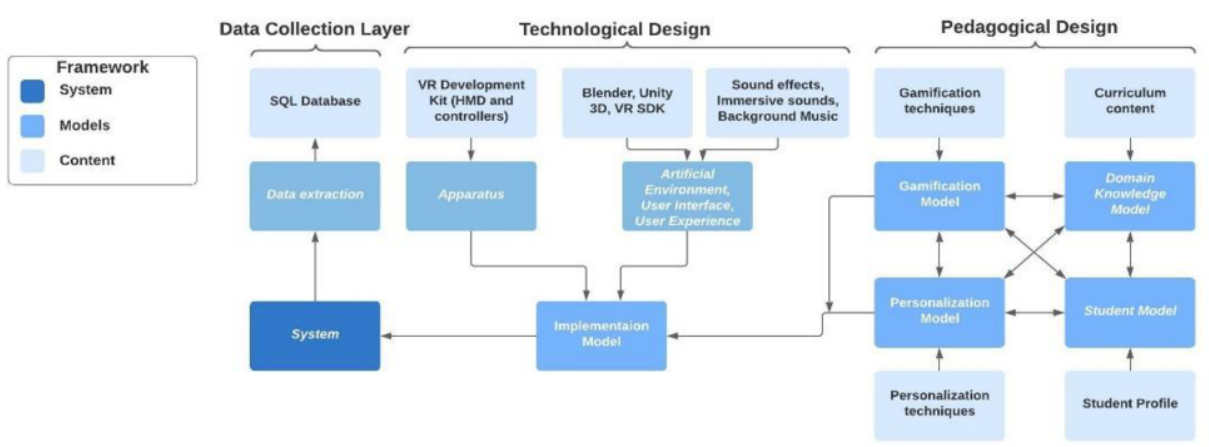

Figure 1. A framework for adaptive Virtual Reality applications using gamification and personalization techniques

\subsection{Pedagogical Design}

The Domain Knowledge Model contains the educational material needed to construct the specific knowledge that defines the curriculum's educational goals.

The Student Model constructs the student's profile, which is in charge of assisting the learning process. It is provided by a system that is designed to take into account student-related factors such as cognitive states, behavioral patterns, preferences, and 
prior knowledge related to the subject and provide relevant feedback and recommendations as the procedure progresses. This could be accomplished through data collection via questionnaires and surveys, or through an in-app procedure similar to those used in commercial games in the form of mini-games when the application first launches as the first task to complete.

The Gamification Model consists of the mechanisms that the system employs to motivate students to use the application and remain engaged throughout the learning process. It aims to increase student retention by providing an enjoyable experience through mechanisms similar to those found in commercial video games.

The Personalization Model works in tandem with the Gamification Model and Student Model to create a tailored learning experience that incorporates data input from student assessments during the Student Model phase, as well as gamification techniques that transform the learning environment into a more exciting and fun learning process that is tailored to students' competencies, skills, and preferences.

\subsection{Technological Design}

The Artificial Environment is where the student participates in the active learning. It is a three-dimensional virtual environment in which students can interact with virtual objects that have been created and configured to allow them to experience life-like situations. The environment consisted of $3 \mathrm{D}$ visual representations, interactions, User Interface, and User experience using the game development platform Unity3D or Unreal Engine that incorporate the Virtual Reality SDK provided by the Head Mounted Display Hardware Development Kit such as Oculus SDK or VIVE Wave SDK. Blender is a software that allows users to create 3D models and animations and offers the option to import them in the game development platform. Furthermore, in order to achieve high levels of immersion and provide students with a flow experience, the systems must produce immersive sound effects and background music.

A Head Mounted Display (HMD) hardware is required for this framework to allow the user to experience three-dimensional visualizations and to serve as a mediator for interactions between the user and the virtual environment and its virtual objects. Since contemporary HMDs use advanced controllers and hand gesture recognition systems, the HTC Vive and Oculus HMDs series are sufficient to meet the requirements of this framework. They also provide high-fidelity visualizations because advanced displays are used.

\subsection{Data Collection Layer}

Data extraction will occur after the required data has been collected as a result of the user's interactions with any virtual elements within the virtual environment. A database will gather all of the required grouped data for further analysis.

\section{Conclusion}

This paper describes a novel framework for designing adaptive, fully immersive Virtual Reality Learning Environments with gamified techniques and aims to become the blueprint for future researches and applications. Taking into account students' cognitive diversity and characteristics, the design of this framework aims to optimize the learning 
experience by transforming it into an effective, appealing, and enjoyable educational approach, as well as creating a tailored educational interactive environment that can be automatically adjusted to the students' profile.

\section{References}

[1] A. Krouska, C. Troussas and M. Virvou, Social networks as a learning environment: Developed applications and comparative analysis, 2017 8th International Conference on Information, Intelligence, Systems \& Applications (IISA) (2017), 1-6. doi: 10.1109/IISA.2017.8316430.

[2] C. Troussas, M. Virvou, J. Caro, K. J. Espinosa, Language Learning Assisted by Group Profiling in Social Networks, International Journal of Emerging Technologies in Learning (IJET) 8(3) (2013), 35.

[3] G. Makransky, R. E. Mayer, N. Veitch, M. Hood, K. B. Christensen, H. Gadegaard, Equivalence of using a desktop virtual reality science simulation at home and in class, Plos One 14(4) (2019), e0214944.

[4] B. Haßler, L. Major, S. Hennessy, Tablet use in schools: a critical review of the evidence for learning outcomes, Journal of Computer Assisted Learning 32(2) (2015), 139-156. doi: 10.1111/jcal.12123.

[5] C. Papakostas, C. Troussas, A. Krouska, C. Sgouropoulou, User acceptance of augmented reality welding simulator in engineering training, Education and Information Technologies (2021).

[6] C. Troussas, Krouska A., Virvou M., Using a Multi Module Model for Learning Analytics to Predict Learners' Cognitive States and Provide Tailored Learning Pathways and Assessment, In: Virvou M., Alepis E., Tsihrintzis G., Jain L. (eds) Machine Learning Paradigms. Intelligent Systems Reference Library, vol 158. Springer (2020). Cham. https://doi.org/10.1007/978-3-030-13743-4_2

[7] R. Lindgren, M. Tscholl, S. Wang, E. Johnson, Enhancing learning and engagement through embodied interaction within a mixed reality simulation, Computers \& Education 95 (2016), 174-187.

[8] C. Papakostas, C. Troussas, A. Krouska, C. Sgouropoulou, Exploration of Augmented Reality in Spatial Abilities Training: A Systematic Literature Review for the Last Decade, Informatics in Education 20 (2021), 107-130. https://doi.org/10.15388/infedu.2021.06

[9] F. A. Monita, J. Ikhsan, Development Virtual Reality IPA (VR-IPA) learning media for science learning, Journal of Physics: Conference Series 1440 (2020), 012103.

[10] J. R. Brown, I. Kuznetcova, E. K. Andersen, N. H. Abbott, D. M. Grzybowski, C. D. Porter, Implementing Classroom-Scale Virtual Reality into a Freshman Engineering Visuospatial Skills Course, 2019 FYEE Conference (2019).

[11] K. Cheng, C. Tsai, A case study of immersive virtual field trips in an elementary classroom: Students' learning experience and teacher-student interaction behaviors, Computers \& Education 140 (2019), 103600. doi: 10.1016/j.compedu.2019.103600

[12] J. Pirker, I. Lesjak, C. Gütl, An Educational Physics Laboratory in Mobile Versus Room Scale Virtual Reality - A Comparative Study, International Journal of Online Engineering (IJOE) 13(08) (2017), 106.

[13] A. Rychkova, A. Korotkikh, A. Mironov, A. Smolin, N. Maksimenko, M. Kurushkin, Orbital Battleship: A Multiplayer Guessing Game in Immersive Virtual Reality, Journal of Chemical Education 97(11) (2020), 4184-4188. https://doi.org/10.1021/acs.jchemed.0c00866

[14] T. Cochrane, S. Cook, S. Aiello, D. Christie, D. Sinfield, M. Steagall, C. Aguayo, A DBR framework for designing mobile virtual reality learning environments, Australasian Journal Of Educational Technology 33(6) (2017), 54-68. doi: 10.14742/ajet.3613

[15] P.Chen, The Design of Applying Gamification in an Immersive Virtual Reality Virtual Laboratory for Powder-Bed Binder Jetting 3DP Training, Education Sciences 10(7) (2020), 172.

[16] C. Hung, I. Huang, G. Hwang, Effects of digital game-based learning on students' self-efficacy, motivation, anxiety, and achievements in learning mathematics, Journal of Computers In Education 1(23) (2014), 151-166. doi: 10.1007/s40692-014-0008-8

[17] A. S. Wilson, J. O'Connor, L. Taylor, D. Carruthers, A 3D virtual reality ophthalmoscopy trainer, The Clinical Teacher 14(6) (2017), 427-431. https://doi.org/10.1111/tct.12646

[18] K. Zhang, J. Suo, J. Chen, X. Liu, L., Gao Design and Implementation of Fire Safety Education System on Campus based on Virtual Reality Technology, Federated Conference on Computer Science and Information Systems (FedCSIS) (2017), 1297-1300. 10.15439/2017F376.

[19] P. Häfner, V. Häfner, J. Ovtcharova, Teaching Methodology for Virtual Reality Practical Course in Engineering Education, Procedia Computer Science 25 (2013), 251-260. doi: 10.1016/j.procs.2013.11.031 
[20] A. P. Johnston, J. Rae, N. Ariotti, B. Bailey, A. Lilja, R. Webb, C. Ferguson, S. Maher, T. P. Davis, R. I. Webb, J. McGhee, R. G. Parton, Journey to the centre of the cell: Virtual reality immersion into scientific data, Traffic 19(2) (2017), 105-110. https://doi.org/10.1111/tra.12538

[21] T. Barrett, M. Hegarty, Effects of interface and spatial ability on manipulation of virtual models in a STEM domain, Computers in Human Behavior 65 (2016), 220-231. doi: 10.1016/j.chb.2016.06.026

[22] C. Rosa-Jimenez, F. Ventura-Blanch, A transversal methodology for the implementation of virtual reality in Architecture learning, Headache (2020), 1347-1354. 10.4995/HEAd20.2020.11274.

[23] M. A. Z. Megat Zakaria, H. Abuhassna, Virtual Reality Acceptance in Classrooms: A Case Study in Teaching Science, International Journal of Advanced Trends in Computer Science and Engineering 9(2) (2020), 1280-1294. https://doi.org/10.30534/ijatcse/2020/58922020

[24] D. Becerra, J. Herrera Quispe, R. Aceituno, G. Vargas, F. Zamora, J. Mango, G. Anccasi Figueroa, A. Vizcarra, J. Chana, Evaluation of a Gamified 3D Virtual Reality System to Enhance the Understanding of Movement in Physics, Proceedings of the 9th International Conference on Computer Supported Education - Volume 1: CSEDU (2017), 395-401. 10.5220/0006328003950401.

[25] W. Yahaya, A. Ahmad, The effectiveness of signaling principle in virtual reality courseware towards achievement of transfer learning among students with different spatial ability, AIP Conference Proceedings 1891 (2017), 020144. 10.1063/1.5005477

[26] E. Surer, M. Erkayaoğlu, Z.N. Öztürk, et al. , Developing a scenario-based video game generation framework for computer and virtual reality environments: a comparative usability study, J Multimodal User Interfaces (2020). https://doi.org/10.1007/s12193-020-00348-6

[27] O. A. Meyer, M. K. Omdahl, G. Makransky, Investigating the effect of pre-training when learning through immersive virtual reality and video: A media and methods experiment, Computers \& Education 140 (2019), 103603. https://doi.org/10.1016/j.compedu.2019.103603

[28] S.-C. Wu, W.-H. Hsiang, The study of the communication effect on emotional design in virtual reality technology using VR application - Facebook spaces as example, Towards Sustainable Technologies and Innovation - Proceedings of the 27th Annual Conference of the International Association for Management of Technology IAMOT 2018. https://www.scopus.com/inward/record.uri?eid=2-s2.085085666893\&partnerID=40\&md5=3d2636a012138e1dd890786055197e74

[29] W. Wizaka, G. Suharjanto, W. Wangidjaja, The new teaching method using virtual reality technology in building technology subject, IOP Conference Series: Earth and Environmental Science 426 (2020), 012080. https://doi.org/10.1088/1755-1315/426/1/012080

[30] J. Calvert, R. Abadia, Impact of immersing university and high school students in educational linear narratives using virtual reality technology, Computers \& Education 159 (2020), 104005.

[31] J. Ikhsan, K. H. Sugiyarto, T. N. Astuti, Fostering Student's Critical Thinking through a Virtual Reality Laboratory, International Journal of Interactive Mobile Technologies (IJIM) 14(08) (2020), 183.

[32] S. Al Kork, T. Beyrouthy, Interactive Virtual Reality Educational Application, Advances in Science, Technology and Engineering Systems Journal 3 (2018), 72-82. 10.25046/aj030409

[33] J. Varela-Aldás, G. Palacios-Navarro, R. Amariglio, I. García-Magariño, Head-Mounted Display-Based Application for Cognitive Training, Sensors 20(22) (2020), 6552. https://doi.org/10.3390/s20226552

[34] A.Domínguez, J. Saenz-de-Navarrete, L. de-Marcos, L. Fernández-Sanz, C. Pagés, J. Martínez-Herráiz, Gamifying learning experiences: Practical implications and outcomes, Computers \& Education 63(2013), 380-392. doi: 10.1016/j.compedu.2012.12.020

[35] J. Bendeck Soto, D. Toro Ocampo, L. Beltrán Colon, A. Valencia Oropesa, Perceptions of ImmerseMe Virtual Reality Platform to Improve English Communicative Skills in Higher Education, International Journal Of Interactive Mobile Technologies (Ijim) 14(07) (2020), 4. doi: 10.3991/ijim.v14i07.12181

[36] A. Klašnja-Milićević, B. Vesin, M. Ivanović, Z. Budimac, E-Learning personalization based on hybrid recommendation strategy and learning style identification, Computers \& Education 56(3) (2011), 885899. https://doi.org/10.1016/j.compedu.2010.11.001

[37] T. Srimadhaven \& AV. Chris, N. Harshith, S. Jessenth, S. Shabari, M. Priyaadharshini, Learning Analytics: Virtual Reality for Programming Course in Higher Education, Procedia Computer Science 172 (2020), 433-437. 10.1016/j.procs.2020.05.095.

[38] A. Krouska, C., Troussas, M. Virvou, Computerized Adaptive Assessment Using Accumulative Learning Activities Based on Revised Bloom's Taxonomy, In: Virvou M., Kumeno F., Oikonomou K. (eds) Knowledge-Based Software Engineering: 2018. JCKBSE 2018. Smart Innovation, Systems and Technologies, vol 108. Springer (2019). Cham. https://doi.org/10.1007/978-3-319-97679-2_26 